Nursing \& Healthcare International Journal
ISSN: $2575-9981$

\title{
Volume 3 Issue 4
}

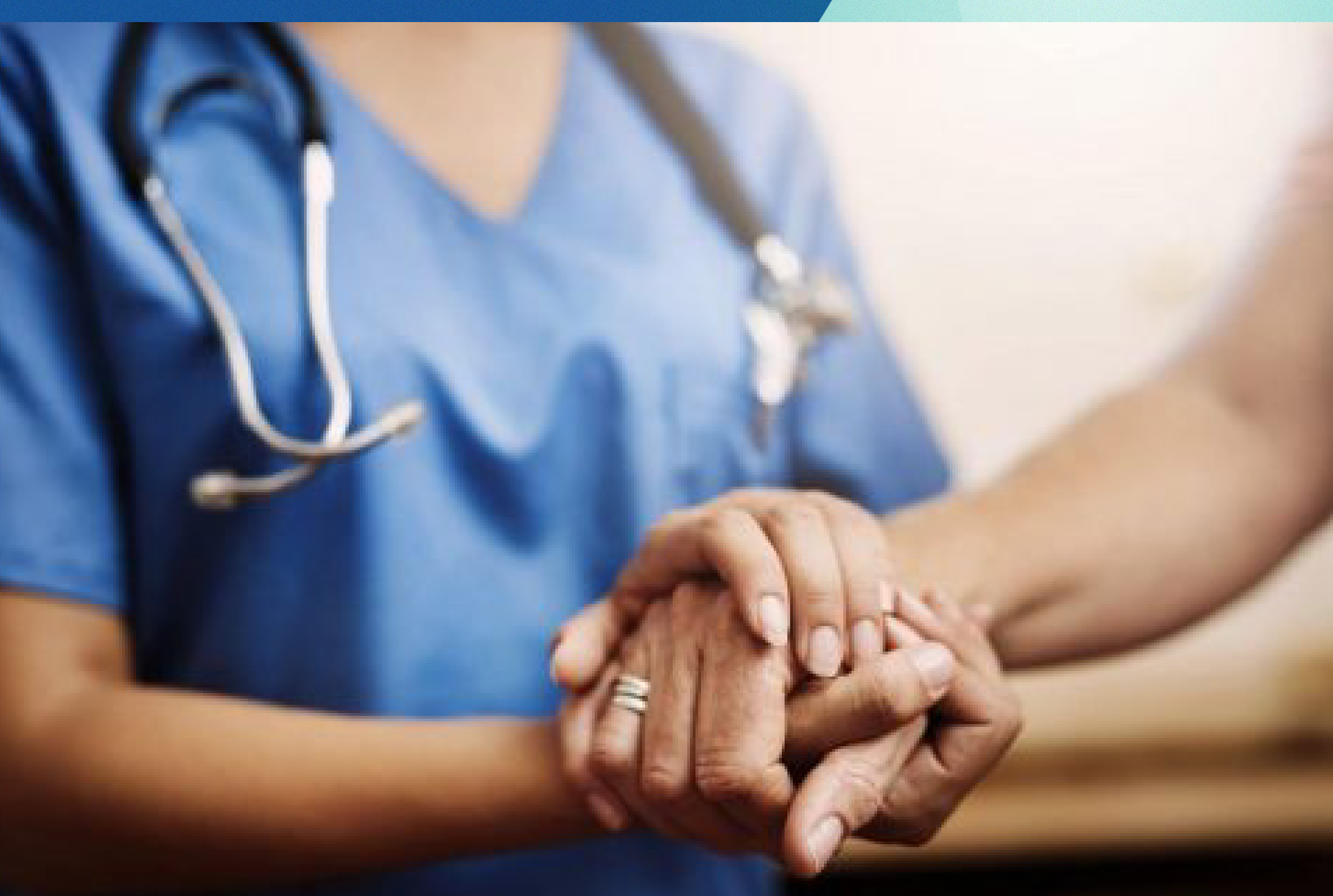

Medwin Publishers 


\title{
Effects of Earplugs and Eye Masks on the Onset of Delirium in a Neuro-Critical Care Unit, Egypt
}

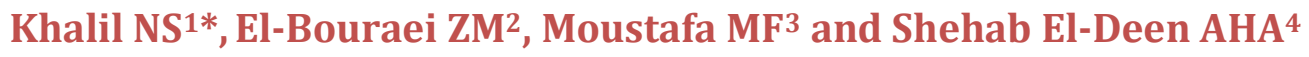 \\ 1Department of Critical Care and Emergency Nursing, Faculty of Nursing, Cairo \\ University, Egypt \\ 2Department of Critical Care and Emergency Nursing, Faculty of Nursing, Mansoura \\ University, Egypt \\ ${ }^{3}$ Department of Neurosurgery, El Sahel Teaching Hospital, Egypt
}

\section{Research Article}

Volume 3 Issue 4

Received Date: July 29, 2019

Published Date: August 21, 2019

DOI: $10.23880 /$ nhij-16000196

*Corresponding author: Nahla Khalil, Department of Critical Care and Emergency Nursing, Faculty of Nursing, Cairo University, Egypt, Tel: 1066225938; Email: nahlakhalil28@yahoo.com

\begin{abstract}
Background: Delirium is a common syndrome in critical care settings. It is caused by a disturbance of the cognitive function in the brain. Non-pharmacological measures such as wearing earplugs and eye Masks could be beneficial in the prevention of delirium by creating relaxing effects.
\end{abstract}

Aim of this Study: was to assess the use of earplugs and eye masks on the onset of delirium during the night in NeuroCritical Care Units (NCCUs).

Material and Methods: Two groups Quasi -experimental research design was used to carry out the research included 66 critically ill adult patients. Random Assignment was performed by the nurse researcher using a computer program. Eligible patients had an expected ICU length of stay more than 24 hours, were arabic language speakers and got Glasgow coma scale of at least 14 . The underlying intervention in the study group included patients' who wear both earplugs and eye masks during the night for three consecutive days. The control group patients were sleeping without earplugs and eye masks during the night. Patients' characteristics, environmental risk factors for delirium and the Delirium Screening and Richmond Agitation and Sedation Scale (RASS) were utilized in data collection.

Results: High significant statistical difference was found between the study and control groups regarding the presence of delirium on the third day $(\mathrm{X} 2=62.118, \mathrm{p}=0.000)$. Moreover, significant differences were found regarding agitation, sedation, and alertness $(\mathrm{X} 2=40.480, \mathrm{p}=0.000)$. So, the study group demonstrated improvement in their Glasgow Coma Score (GCS) than the control group.

Conclusion: Using earplugs and eye masks at night in NCCU have decreased the onset of delirium and improving patients' GCS.

Recommendation: Replication of the study on a large probability sample selected from different NCCUs in Egypt. Furthermore, evidence-based protocols for using earplugs and eye masks should be available in prescribed form in different ICU settings.

Keywords: Delirium; Earplugs; Eye masks; Neuro-Critical Care Units 


\section{Nursing \& Healthcare International Journal}

\section{Introduction}

Delirium is a prevalent neuropsychiatric syndrome characterized by a change in conscious level and disturbance of cognitive functioning. The occurrence of delirium in critical care settings varies from $10 \%$ to $31 \%$, as decided by patients' characteristics and the evaluation methods utilized [1]. In ICU settings, mixed and hypoactive delirium is the most familiar types, and are badly diagnosed if consistent monitoring is not done. Few critically ill patients experience unusual hyperactive delirium [2].

Up till now, pharmacological researches have not been exhibited to be successful for the therapy of delirium but non-pharmacological approaches have shown some consensus [3]. They are viewed as the best remedial measures because the advised drugs in controlling the manifestation of delirium may increase or lengthen its progression [4].

There are many non-pharmacological therapeutic measures for the prevention of delirium. First; make the best effective use of sleep-wake pattern during daytime by extended subjection to daylight and keep curtains opened. On the other hand, during night, light and sounds must be reduced [5]. Second; Re-orientating the critically ill patients by describing the place, patients' identification, health care providers around the patient, and their duty, visible clock, and prevent frequent changing of patients' rooms [6].

Third; use of communication aids such as eyeglasses, hearing aids, and dentures when needed, moving the patient if health condition gives the patients opportunity sitting out of bed for eating if allowed, and prevent application of physical restraints are useful measures in preventing delirium [7]. Other useful measures for avoiding the incidence of delirium include environmental and behavioral modifications that aimed to make the patient's environment as safe $[8,9]$.

\section{Aim of the Study}

The aim of the present study was to evaluate effects of earplugs and eye masks on the onset of delirium in neurocritical care unit.

\section{Research Hypothesis}

H1: Use of earplugs and eye masks during the night reduce onset of delirium in the Neuro Critical Care Unit.

\section{Subjects and Methods}

\section{Research Design}

A quasi-experimental research design was used to conduct this study. So, the patients are assigned to study or control groups, they are considered the gold standard for assessing causality and, are a first choice for most intervention research [10].

\section{Study Setting}

The study was carried out at the neuro critical care unit in Mansoura New General Hospital. That unit admits almost 40 patients monthly from Mansoura city and adjacent cities around Mansoura city.

\section{Subjects}

A convenient sample of 66 patients who admitted to neuro-critical care unit was enrolled in this study. The exclusion criteria included who were younger than 18 years old, their Glasgow Coma Scale at least 10, having ear or eye injuries on admission, complained of hearing or vision problems, having delirium, confusion or sleeping problems on admission, and patients who were under sedation or narcotic drugs during the study.

\section{Calculation of Sample Size}

Sample size was calculated depending on the following measurements; Population size (80 patients, all patients admitted to Neuro Critical Care Unit (NCCU), Expected frequency (20\%), Margin of error (5\%), confidence coefficient (95\%), and minimum sample size (61 patients). The sample was estimated according to Epi Info 7 sample size estimation program 2013 using the following parameter:

$$
\begin{aligned}
& N=(Z 1-\alpha / 2+Z 1-\beta)^{2} \sigma 1 \sigma 2 / \delta^{2} \\
& Z 1-\alpha / 2=1.96 \\
& Z 1-\beta=0.842
\end{aligned}
$$

$\sigma 1 \sigma 2=$ SD for each group

$\delta=$ Expected difference to be detected between 2 groups

$\alpha=$ Level of acceptability of a false positive result (level of significance $=0.05$ )

$\beta=$ Level of acceptability of a false negative result $(0.20)$

$1-\beta=$ power $(0.80)$

The sample size was 66 patients. These 66 patients were divided equally and equitably into the study and control groups. Each group included 33 patients. The control group included patients who didn't wear the 


\section{Nursing \& Healthcare International Journal}

earplugs or the eye masks at night during sleep, while the study group included patients who wore the earplugs and eye masks at nights. Both groups continued participation in the study for at least 3 nights.

\section{Tools of Data Collection}

\section{Tool I: Patients' Characteristics and Environmental Risk Factors for Delirium}

This tool was elaborated by the researcher: it included demographic data, health-relevant data and environmental risk factors for delirium such as light, sound, and alarms.

\section{Tool II: Delirium Assessment Tools}

This tool was selected from Patel J, et al. [11]. It involved two parts; Delirium Screening Checklist and Richmond Agitation and Sedation Scale. First; The delirium screening checklist that aimed to assess the presence of delirium. It included 8 symptoms that are evaluated over 8-24 hour duration. The Patients are obtained one score for each symptom that exhibits during the time period and zero score if symptom did not exhibit. A score more than 4 points out the presence of delirium The eight symptoms of delirium included; level of consciousness, inattention, disorientation, hallucinations/ delusions/ psychosis, psychomotor agitation or retardation, inappropriate speech or mood, sleep/wake cycle disturbances, and fluctuation.

Second; The Richmond agitation and sedation Scale aimed to assess sedation and agitation in critically ill patients. It is a scale included 10 point with 4 levels of anxiety or agitation ranging from +1 to +4 , one level to denote a calm and alert state (0) and 5 levels to assess the level of sedation ranging from -1 to -5 . A score of +4 indicates that patient openly violent and is considered dangerous to the caring staff. A score of -4 indicates that the patient is unresponsive to verbal stimulation and finally, coming in unarousable states $(-5)$

A score of +1 to +4 denotes the increasing level of agitation and a score of -1 to -5 denotes the increasing level of sedation. Patients with a RASS of -3 or less should have their sedation decreased or modified in order to achieve a RASS of -2 to zero. Patients with a RASS of 2 to 4 are not sedated adequately and should be evaluate for presence of pain, anxiety, or delirium. The primary causes of the agitation should be examined and treated in a suitable manner to reach to a RASS of -2 to zero. In the present study, according to the patients' Glasgow coma scale scores, they were evaluated for the agitation level starting with zero scores, so the minus scores indicated that patients were unconscious.

\section{Validity and Reliability of Tools}

The tools of the study were examined for content validity by five experts in the fields of neuro-Surgery and critical care nursing. The experts' modifications were editing of some sentences Next, the adopted tools were tested for reliability using Cronbach 's Alpha test, to measure the internal consistency of tools that included delirium checklist and Richmond agitation scale $(r=0$. 774).

\section{Protection of Human Rights}

An Ethical approval was obtained from the Research Ethical Committee at Faculty of nursing Mansoura University. As well, an official letter to conduct the study was obtained from the hospital administrative authority was attained. Furthermore, oral consent was obtained from the patients.

\section{Procedure}

The current study was conducted through three phases; assessment, implementation, and evaluation phase.

\section{1-Assessment}

A primary assessment was carried out by the researcher on the first day for all neuro-critical ill patients through collecting data pertinent to patients' characteristics and environmental risk factors for delirium as well as delirium assessment tools to confirm that they don't have delirium and free from exclusion criteria.

\section{2-Implementation}

After completion of the assessment phase, the researcher performed the intervention of wearing earplugs and eye masks among the study groups as follows: Earplugs and eye masks were worn at the same time each night from 10 p. m. to 7 a. m. for 3 consecutive nights. Earplugs were easily worn. During which, the nurse rolled earplugs up into a small, thin "snake" with fingers utilizing one or both hands. Later, the nurse pulled the top of ear up and back with the opposite hand to straighten out the ear canal and the rolled-up earplug was slide right in. Next, the earplug was held in with finger, and counted to 20 or 30 out loud while waiting for the plug to expand and fill-in the ear canal during which, the 


\section{Nursing \& Healthcare International Journal}

voice sounded muffled when the plug has made a good seal. After that, the nurse checked the fit when all done. Most of the foam body of the earplug should be within the ear canal. Moreover, the nurse cupped hands tightly over ears properly. If sounds are much more muffled with hands in place, the earplug may not be sealing properly and the nurse took the earplug out and tried again. Finally, the nurse removed the earplug slowly with a twisting motion to gradually break the seal to avoid damage to the eardrum. This is a disposable earplug. It is advised that this earplug not to be used again. Earplugs should be continued clean by removing the earwax and discharges before re-insertion. Soiled ear plugs may be rubbed clean with a moist swab. As well, solutions, disinfectants and chemicals are never used [12].

Concerning wearing of eye masks, they were worn through the following steps: first; the nurse fitted the mask snugly over the head, and the colored side of the mask faces outwards. Second; the strings or elastic bands are positioned properly to keep the mask firmly in place around the head. Third; the nurse wore his/her patient eye mask firmly, and not too tight or not too loose. Finally, the nurse ensured that no light penetrated the patient's eye [13].

Regarding the implementation of traditional procedure of the control group, the nurse performed the following actions as follows; the patients were left all the three nights of the study period without using earplugs or eye masks, and they were observed by the nurse researcher throughout the study period utilizing delirium assessment tools.

\section{3-Evaluation}

Initially, the researcher made a comparison between baseline data between the study group and the control group concerning personal and health relevant data such as Glasgow coma score, length of stay in ICU. As well, the researcher made the comparison between both groups in relation to the presence of delirium. Next, after completion of both interventions in the study and control groups, the researcher carried out again the comparison between both groups to ascertain that the impact of earplugs and eye masks have an impact on the onset of delirium in the study group compared to the control group.

\section{Data Analysis}

Data were analyzed utilizing SPSS version 22. Data were presented in the form of means, frequencies and percentages. Chi square was used for comparison and correlation between quantitative data. Moreover, $t$ test was calculated for comparison of quantitative data between groups.

\section{Results}

Table 1 illustrates the demographic characteristics of the studied sample. It revealed that more than one-third of the studied patients in the study group and the control group, their age varied between 20 - 30 years with the mean age $35.42 \pm 14.02 \& 34.48 \pm 14.04$ respectively.

\begin{tabular}{|c|c|c|c|c|c|}
\hline \multirow{3}{*}{ Variables } & \multirow{2}{*}{\multicolumn{2}{|c|}{$\begin{array}{c}\text { Study group } \\
(n=33)\end{array}$}} & \multirow{2}{*}{\multicolumn{2}{|c|}{$\begin{array}{c}\text { Control group } \\
(n=33)\end{array}$}} & \multirow{3}{*}{ Significant } \\
\hline & & & & & \\
\hline & No & $\%$ & No & $\%$ & \\
\hline \multicolumn{6}{|l|}{ Age (Years) } \\
\hline$<20$ & 3 & 9.1 & 4 & 12.1 & \\
\hline $20-29$ & 11 & 33.3 & 10 & 30.3 & $X^{2}=23.628$ \\
\hline $30-39$ & 7 & 21.2 & 8 & 24.2 & $\mathrm{P}=0.57$ \\
\hline $40-49$ & 8 & 24.2 & 6 & 18.2 & \\
\hline$\geq 50$ & 4 & 12.1 & 5 & 15.2 & \\
\hline Min - Max & \multicolumn{2}{|c|}{$18-72$} & \multicolumn{2}{|c|}{$18-72$} & $t=1.876$ \\
\hline Mean \pm SD & \multicolumn{2}{|c|}{$35.42 \pm 14.02$} & \multicolumn{2}{|c|}{$34.48 \pm 14.04$} & $\mathrm{P}=0.132$ \\
\hline \multicolumn{6}{|l|}{ Gender } \\
\hline Male & 17 & 51.5 & 20 & 60.6 & $X^{2}=0.554$ \\
\hline Female & 16 & 48.5 & 13 & 39.4 & $\mathrm{P}=0.46$ \\
\hline
\end{tabular}

Table 1: Frequency distribution of the studied sample according to their patients' characteristics $(n=66)$. 
Regarding gender, almost half of the study group (51. $5 \%$ ) were male and (48.5\%) were female in the control group. However, no statistically significant differences were found between both groups regarding age and gender $(\mathrm{p}=0.57 \& \mathrm{p}=0.46$ respectively).

\begin{tabular}{|c|c|c|c|c|c|}
\hline \multirow[t]{2}{*}{ Variables } & \multicolumn{2}{|c|}{$\begin{array}{c}\text { Study group } \\
(\mathrm{n}=33)\end{array}$} & \multicolumn{2}{|c|}{$\begin{array}{l}\text { Control group } \\
(n=33)\end{array}$} & \multirow[t]{2}{*}{$\mathbf{X}^{2} ; \mathbf{p}$} \\
\hline & No & $\%$ & $\mathbf{N}$ & $\%$ & \\
\hline \multicolumn{6}{|l|}{ Diagnosis } \\
\hline Acute Sub-dural hemorrhage & 4 & 12.1 & 2 & 6.1 & \multirow{14}{*}{$\mathrm{X} 2=11.986 \mathrm{P}=0.85$} \\
\hline Space Occupying Lesion & 2 & 6.1 & 3 & 9.1 & \\
\hline Depressed skull fracture & 2 & 6.1 & 3 & 9.1 & \\
\hline Lumbar Disc Prolapse & 1 & 3.0 & 1 & 3.0 & \\
\hline Depressed skull fracture + Hemorrhagic Brain Contusion & 3 & 9.1 & 1 & 3.0 & \\
\hline Hemorrhagic Brain Contusion & 3 & 9.1 & 4 & 12.1 & \\
\hline Depressed fracture & 3 & 9.1 & 1 & 3.0 & \\
\hline Pneumocephalus & 4 & 12.1 & 3 & 9.1 & \\
\hline Chronic Sub-Dural Hemorrhage & 1 & 3.0 & 5 & 15.2 & \\
\hline Dorsal fracture & 4 & 12.1 & 3 & 9.1 & \\
\hline Cervical fracture & 2 & 6.1 & 2 & 6.1 & \\
\hline Dorsal tumourse & 1 & 3.0 & 1 & 3.0 & \\
\hline Extra Dural Hemorrhage + Hemorrhagic Brain Contusion & 1 & 3.0 & 1 & 3.0 & \\
\hline Extra Dural Hemorrhage + Pneumocephalus & 1 & 3.0 & 1 & 3.0 & \\
\hline \multicolumn{6}{|l|}{ Length of hospitalization } \\
\hline 3 days & 23 & 69.7 & 11 & 33.3 & \multirow{3}{*}{$\mathrm{X} 2=10.028 \mathrm{P}=0.007^{*}$} \\
\hline 4 days & 10 & 30.3 & 19 & 57.6 & \\
\hline 5 days & 0 & 0.0 & 3 & 9.1 & \\
\hline \multicolumn{6}{|l|}{ Glasgow coma score } \\
\hline $1^{\text {st }}$ day & & & & & \multirow{3}{*}{$\mathrm{X}^{2}=1.158 \mathrm{P}=0.28$} \\
\hline 14 & 6 & 18.2 & 3 & 9.1 & \\
\hline 15 & 27 & 81.8 & 30 & 90.9 & \\
\hline \multicolumn{6}{|l|}{$2^{\text {nd }}$ day } \\
\hline 14 & 1 & 3.0 & 2 & 6.1 & \multirow{2}{*}{$\mathrm{X}^{2}=0.349 \mathrm{P}=0.55$} \\
\hline 15 & 32 & 97.0 & 31 & 93.9 & \\
\hline \multicolumn{6}{|l|}{$3^{\text {rd day }}$} \\
\hline 14 & 0 & 0.0 & 3 & 9.1 & \multirow{2}{*}{$\mathrm{X}^{2}=3.143 \mathrm{P}=0.08$} \\
\hline 15 & 33 & 100.0 & 30 & 90.9 & \\
\hline
\end{tabular}

Table 2: Frequency distribution of the studied sample by their health relevant data $(n=66)$.

- * p-value $\leq 0.05$ (significant). - ** p-value $\leq 0.001$ (highly significant).

Table 2 illustrated the frequency distribution of the studied patients by their health-relevant data. It revealed that $12.1 \%$ of the study group had a diagnosis of acute subdural hemorrhage (ASDH), dorsal fracture and Pneumocephalus. On the other hand, $12.1 \%$ and $15.2 \%$ of patients in the control group had a diagnosis of hemorrhagic brain contusion (HBC) and chronic subdural hemorrhage (CSDH). A statistically significant difference was detected between the study and the control group in relation to the length of hospitalization $(p=0.007)$. So, more than two-thirds of the study group stayed in the hospital for three days (69.7\%) compared to the control group who stayed four days (57.6\%). Regarding the Glasgow Coma Scale scoring over three days, it was found no significant differences between both groups $(\mathrm{X} 2=3.1$; $\mathrm{P}=0.08)$.

Table 3 illustrates the distribution of the studied sample regarding the presence of delirium in the three consecutive days. It revealed that there was a statistically significant difference between the study and control group in the second day $\left(\mathrm{X} 2=4.275 ; \mathrm{P}=0.031^{*}\right)$ with Mean \pm SD $(0.697 \pm 0.683 \& 2.636 \pm 0.783$ respectively $)$. On the other hand, no significant differences were found 
between both groups in the first and third days ( $\mathrm{X} 2=$ 4.208; $\mathrm{P}=0.377$ \& $\mathrm{X} 2=6.085 ; \mathrm{P}=0.133$ respectively) for the first and third days with Mean \pm SD $(1.545 \pm 0.832 \&$ $1.636 \pm 0.962$ respectively) for both the study and control groups in the first day \& $(0.242 \pm 0.502 \& 3.394 \pm 1.297$ respectively) for both the study and control groups in the third day.

\begin{tabular}{|c|c|c|c|c|c|}
\hline \multirow{2}{*}{ Presence of delirium } & \multicolumn{2}{|c|}{ Study group $(n=33)$} & \multicolumn{2}{|c|}{ Control group $(n=33)$} & \multirow{2}{*}{ Significant } \\
\hline & No & $\%$ & No & $\%$ & \\
\hline \multicolumn{6}{|l|}{$1^{\text {st }}$ day } \\
\hline Yes & 0 & 0.00 & 0.0 & 0.00 & $\mathrm{X}^{2}=4.208$ \\
\hline No & 33 & 100 & 33 & 100 & $P=0.37$ \\
\hline Mean \pm SD & \multicolumn{2}{|c|}{$1.545 \pm 0.832$} & \multicolumn{2}{|c|}{$1.636 \pm 0.962$} & \\
\hline \multicolumn{6}{|l|}{$2^{\text {nd }}$ day } \\
\hline Yes & 0.0 & 0.00 & 2 & 6.1 & $\mathrm{X}^{2}=4.275$ \\
\hline No & 33 & 100 & 31 & 93.9 & $\mathrm{P}=0.031^{*}$ \\
\hline Mean \pm SD & \multicolumn{2}{|c|}{$0.697 \pm 0.683$} & \multicolumn{2}{|c|}{$2.636 \pm 0.783$} & \\
\hline \multicolumn{6}{|l|}{$3^{\text {rd day }}$} \\
\hline Yes & 0.0 & 0.00 & 14 & 42.4 & $X^{2}=6.085$ \\
\hline No & 33 & 100 & 19 & 57.6 & $\mathrm{P}=0.133$ \\
\hline Mean \pm SD & \multicolumn{2}{|c|}{$0.242 \pm 0.502$} & \multicolumn{2}{|c|}{$3.394 \pm 1.297$} & \\
\hline
\end{tabular}

Table 3: Frequency distribution of the studied sample regarding the presence of delirium $(n=66)$.

- * p-value $\leq 0.05$ (significant). - ** p-value $\leq 0.001$ (highly significant).

Table 4 showed the Comparison between the study and control group regarding environmental risk factors for delirium. It revealed a high significant statistical difference between the study and control groups. So the study group who wore the eye mask and earplug perceived and reported lower noise, light, alarms, and health team members' phones when compared to the control group patients.

\begin{tabular}{|c|c|c|c|c|}
\hline \multirow{2}{*}{ Variables } & Study group $(n=33)$ & Control group $(n=33)$ & \multirow{2}{*}{ t-value } & \multirow{2}{*}{ p-value } \\
\hline & Mean \pm SD & Mean \pm SD & & \\
\hline Noise & $7.64 \pm 0.70$ & $2.88 \pm 0.60$ & -29.670 & $0.000^{* *}$ \\
\hline Light & $7.61 \pm 0.83$ & $2.70 \pm 0.59$ & -27.834 & $0.000^{* *}$ \\
\hline Alarms & $8.0 \pm 0.87$ & $2.61 \pm 0.56$ & -30.114 & $0.000^{* *}$ \\
\hline O2 finger probe & $4.18 \pm 0.68$ & $3.15 \pm 0.62$ & -6.425 & $0.000^{* *}$ \\
\hline Talking & $3.36 \pm 0.55$ & $2.21 \pm 0.42$ & -9.613 & $0.000 * *$ \\
\hline Nurses and doctors phones & $3.06 \pm 0.56$ & $-2.00 \pm 0.25$ & -10.00 & $0.000^{* *}$ \\
\hline Visible Wall Clock & 0 & 0 & 0 & NA \\
\hline
\end{tabular}

Table 4: Comparison between study and control group regarding environmental risk factors for delirium.

Table 5 illustrated the level of agitation and sedation among the study and the control group, it revealed a statistically significant difference between both groups in relation to the level of agitation and sedation in three days where $(\mathrm{p}=0.000)$; so, the participants in the control group had more agitation than the participants in the study group in the second and the third day $(100.0 \% \& 63.6$ respectively) \& (100. $0 \%$ \& $24.2 \%$ respectively), while the participants in the study group had agitation more than the control group on the first day only $(100.0 \& 42.2$ respectively). 


\section{Nursing \& Healthcare International Journal}

\begin{tabular}{|c|c|c|c|c|c|}
\hline \multirow{2}{*}{ Variables } & \multicolumn{2}{|c|}{$\begin{array}{l}\text { Study group } \\
(n=33)\end{array}$} & \multicolumn{2}{|c|}{$\begin{array}{c}\text { Control group } \\
(n=33)\end{array}$} & \multirow{2}{*}{ Significant } \\
\hline & No & $\%$ & No & $\%$ & \\
\hline \multicolumn{6}{|c|}{ Level of agitation and sedation } \\
\hline \multicolumn{6}{|l|}{ 1st day: } \\
\hline Agitation & 33 & 100.0 & 19 & 42.2 & \multirow{3}{*}{$\mathrm{t}=4.865 \mathrm{p}=0.000^{* *}$} \\
\hline Sedation & 0 & 0.0 & 0 & 0.0 & \\
\hline Alert and calm & 0 & 0.0 & 14 & 57.6 & \\
\hline \multicolumn{6}{|l|}{$2^{\text {nd }}$ day: } \\
\hline Agitation & 21 & 63.6 & 33 & 100.0 & \multirow{3}{*}{$\mathrm{t}=-4.276 \mathrm{p}=0.000^{* *}$} \\
\hline Sedation & 0 & 0.0 & 0 & 0.0 & \\
\hline Alert and calm & 12 & 36.4 & 0 & 0.0 & \\
\hline \multicolumn{6}{|l|}{ 3rd day: } \\
\hline Agitation & 8 & 24.2 & 33 & 100.0 & \multirow{3}{*}{$\mathrm{t}=-10.000 \mathrm{p}=0.000^{* *}$} \\
\hline Sedation & 0 & 0.0 & 0 & 0.0 & \\
\hline Alert and calm & 25 & 75.8 & 0 & 0.0 & \\
\hline Significant & \multicolumn{2}{|c|}{$\mathrm{X}^{2}=40.480 \mathrm{P}=0.000^{* * *}$} & \multicolumn{2}{|c|}{$\mathrm{X}^{2}=19.521 \mathrm{P}=0.000^{* * *}$} & \\
\hline
\end{tabular}

Table 5: Frequency distribution of the studied sample regarding their level of agitation and sedation ( $\mathrm{n}=66)$.

- * p-value $\leq 0.05$ (significant). - ** p-value $\leq 0.001$ (highly significant).

Table 6 illustrated the correlation between selected environmental factors (Noise, Light, and Alarms) and the Presence of delirium along the three days of the Study. It revealed no significant correlation what so ever between the light, noise, alarms and the presence of delirium among the study group subjects in the three days. On the other hand, a significant positive correlation was found between noise, light, and presence of delirium in the second and third days only, but no significant correlation between alarms and presence of delirium in three days.

\begin{tabular}{|c|c|c|c|c|c|c|}
\hline \multirow[t]{2}{*}{ Environmental concerns } & \multicolumn{3}{|c|}{$\begin{array}{c}\text { Delirium } \\
\text { (Study group) }\end{array}$} & \multicolumn{3}{|c|}{$\begin{array}{c}\text { Delirium } \\
\text { (Control group) }\end{array}$} \\
\hline & $1^{\text {st }}$ day & $2^{\text {nd }}$ day & $3^{\text {rd }}$ day & $1^{\text {st }}$ day & $2^{\text {nd }}$ day & $3^{\text {rd day }}$ \\
\hline \multicolumn{7}{|c|}{ Noise } \\
\hline $\mathrm{r}$ & -0.114 & 0.06 & -0.107 & 0.308 & $0.493^{* *}$ & $0.680^{* *}$ \\
\hline $\mathrm{P}$ value & 0.528 & 0.74 & 0.554 & 0.081 & 0.004 & 0 \\
\hline \multicolumn{7}{|c|}{ Light } \\
\hline $\mathrm{r}$ & -0.163 & -0.002 & -0.061 & 0.286 & $0.544^{* *}$ & $0.790^{* *}$ \\
\hline P value: & 0.364 & 0.99 & 0.735 & 0.107 & 0.001 & 0 \\
\hline \multicolumn{7}{|c|}{ Alarms } \\
\hline $\mathrm{r}$ & 0.209 & 0.005 & 0.017 & 0.075 & 0 & 0.306 \\
\hline P value: & 0.244 & 0.978 & 0.925 & 0.678 & 1 & 0.083 \\
\hline
\end{tabular}

Table 6: Correlation between selected environmental factors (Noise, Light, And Alarms) and the Presence of delirium along the three days of the Study $(n=66)$.

- * p-value $\leq 0.05$ (significant). - ** p-value $\leq 0.001$ (highly significant).

\section{Discussion}

The present study aimed to examine the impact of earplugs and eye masks on the onset of delirium in neurocritical care unit. Although the sample was smaller than required, worthy recognition about impact of earplugs and eye masks on occurrence of delirium were detected. The current study revealed highly significant statistical differences between the study and control group regarding the presence of delirium in the second and third days. So, the study group subjects who wore the eye mask and earplugs showed decreased mean of 


\section{Nursing \& Healthcare International Journal}

delirium scores. This finding may have relevant to the fact that using earplugs and eye masks improves sleep of the patients in critical care settings to reduce the environmental noise that arise from many causes, including phones sounds, talking, and equipment sounds originated from suction machines and mechanical ventilation. One other possible explanation for that finding wearing mask and ear plug prevent sensory stimulation and prevent the release of adrenalin by the sympathetic nervous system, and these effects makes the patient relaxing and falling asleep and decrease the onset of delirium. Similar to the current study Van Rompaey, et al. [14], who examined the effect of earplugs use in the night on the occurrence of delirium and sleep perception proved that the use of earplugs during the night was favorable, in the study group and few patients revealed delirium. Furthermore, a recent meta-analysis proposed that the utilization of earplugs and an eye mask was accompanied by a significant decrease of delirium $[15,16]$.

In the same context, the current finding is consistent with Mahran KSG, et al. [17] who investigated the effect of night light on delirium occurrence in post-operative cardiac patients utilizing eye mask revealed a significant decrease in delirium occurrence in experimental group than routine care group. These results were related to the improvement in sleeping quality in the experimental group than routine care group. Moreover Patel J, et al. [11] examined the effect of multi-component bundle on sleeping and delirium. One component of the bundle was wearing eye mask and earplugs. That study revealed decreased incidence of delirium and declined number of awakenings caused by care activities overnight. On the other hand, previous studies have illustrated that many patients found earplugs and even an eye mask very uncomfortable [18] with compliance average rate $13 \%$ $[15,17]$. Patients suffered earplugs not settled in place and causing ears sores or experienced anxiety when they did not hear any surrounding noise [18-20]. As well, Patients complained that eye masks make them flushed, sweaty and were too firm and tense causing a feeling of fear of being in enclosed places. (18). As a result, patients' may be opposed to use earplugs or an eye mask [21]. This is definitely true in that patients of the study group who wore earplugs only part of night had even worse sleep quality than patients in the control group.

Concerning the level of agitation and sedation among the study and the control group, there was a statistically significant difference in relation to the level of agitation and sedation in the three days. So, the participants in the control group had more agitation than the study group in the second and the third days. Our finding is consistent with Joseph A, et al. [22] who stated that the night use of earplugs and eye masks reduced the incidence of confusion in a study of 69 ICU patients, and these approaches were effective and minimally invasive solution. As well, our finding is consistent with Van Rompaey, et al. [14] who investigated the impact of earplugs in the night on the occurrence of delirium and sleep perception and found the cognitive disruption was postponed compared to the patients sleeping without earplugs and more patients noted a better sleep after sleeping with earplugs in the ICU. In the same context, our finding is agreed with Kamdar B, et al. Van Rompaey B, et al. Inouye $S$, et al. [23-25] who mentioned that noise ICU environment and continuous bright lights are irritable and unsatisfactory because evidence illustrates that such stimuli can result in psychological and physiologic problems such as agitation, confusion, and delirium follow the stimuli of sleep interrupting noises.

When investigating the correlations between the environmental ICU factors such as noise, light, and alarms on onset of delirium along the three consecutive night times, it was found that there was strong positive correlation between presence of delirium, noise and light among the control group patients in the second and third days of the study. On the other hand, no correlations were found between the environmental factors and delirium among the study group. This finding indicated that exposure to excessive noise levels is a contributing factor in the development of intensive care unit delirium. On the same line, our findings were supported by Xie J, et al. [26] who researched a review of 11 noise studies and found that noise was the most significant factor for sleep disturbance in nearly half of the studies and resulting in occurrence of delirium. The most common sources of noise were staff talking and alarm sounds particularly those with high frequencies.

Similarly, Ghaeli P, et al. [27], stated that noise and light are two environmental factors that disturb sleep and might increase delirium symptoms. Therefore, they recommended the reduction of noises and lights in the patients' environment to improve patients' sleep and thus reduce onset of delirium. On the other hand -Estrup S, et al. [28], didn't find an association between circadian light and development of delirium.

Finally, the present study found no significant correlations between presence of delirium and alarms among both the study and control group in three consecutive night times of the study. That finding was in contrast with Park M, et al. [29] who found that alarms represented $30 \%$ of the total surrounding noise in 


\section{Nursing \& Healthcare International Journal}

intensive care unit and they contributed in occurrence of delirium.

\section{Conclusion}

These stressors were reduced significantly by the earplugs and eye masks at night and using them decreased the occurrence of delirium and improved quality of sleep in neuro-critical care unit.

\section{Recommendations}

Replication of the study on a large probability sample from different geographical areas of Egypt and in general intensive care units is recommended. Furthermore, studying the barriers facing patients in critical care units and lead them to delirium. Also, evidence-based delirium care protocols or bundles should be integrated in intensive to improve patients' quality of care.

\section{Limitation of the Study}

- The sample was drawn from one hospital in one geographical area in the Arab republic of Egypt that restricts generalization of findings.

- Some patients refused to wear both earplugs and eye masks because they felt uncomfortable, due to experiencing pain after wearing them

\section{References}

1. Rivière J, van der Mast RC, Vandenberghe J, Van Den Eede F (2019) Efficacy and tolerability of atypical antipsychotics in the treatment of delirium: a systematic review of the literature. Psychosomatics 60(1): 18-26.

2. Cavaliere F (2016) Postoperative Delirium. In Topical Issues in Anesthesia and Intensive Care, pp: 155-163.

3. Bannon L, McGaughey J, Clarke M, McAuley DF, Blackwood B (2016) Impact of non-pharmacological Studies on prevention and treatment of delirium in critically ill patients: protocol for a systematic review of quantitative and qualitative research. Syst rev 5(1): 75.

4. Gilmore JP, Weigel KA (2017) Delirium. In Dementia in Nursing Homes, pp: 191-201.

5. Bush SH, Tierney S, Lawlor PG (2017) Clinical Assessment and Management of Delirium in the Palliative Care Setting. Drugs 77(15): 1623-1643.
6. Rosen T, Connors S, Clark S, Halpern A, Stern ME, et al. (2015) Assessment and management of delirium in older adults in the emergency department: literature review to inform development of a novel clinical protocol. Advanced emergency nursing journal 37(3): 183-196.

7. Teale EA, Siddiqi N, Clegg A, Todd OM, Young J (2017) Non-pharmacological Studies for managing delirium in hospitalized patients. Cochrane Database of Systematic Reviews.

8. Abraha I, Trotta F, Rimland JM, Cruz-Jentoft A, Lozano-Montoya I, et al. (2015) Efficacy of nonpharmacological Studies to prevent and treat delirium in older patients: a systematic overview. The SENATOR project ONTOP Series. Plos One 10(6): e0123090.

9. Schwartz AC, Fisher TJ, Greenspan HN, Heinrich TW (2016) Pharmacologic and nonpharmacologic approaches to the prevention and management of delirium. Int J Psychiatry Med 51(2): 160-170.

10. Campbell DT, Stanley JC (2015) Experimental and quasi-experimental designs for research. Ravenio Books.

11. Patel J, Baldwin J, Bunting P, Laha S (2014) The effect of a multicomponent multidisciplinary bundle of interventions on sleep and delirium in medical and surgical intensive care patients. Anaesthesia 69(6): 540-549.

12. Delfino B, Delfino G, Skeuse K, Dowd P (2018) US Patent Application No. 15/719, 862.

13. Bruder MH (2017) US Patent Application No. 15/443, 199.

14. Van Rompaey B, Elseviers MM, Van Drom W, Fromont V, Jorens PG (2012) The effect of earplugs during the night on the onset of delirium and sleep perception: a randomized controlled trial in intensive care patients. Crit Care 16(3): R73.

15. Litton E, Carnegie V, Elliott R, Webb SA (2016) The efficacy of earplugs as a sleep hygiene strategy for reducing delirium in the ICU: a systematic review and meta-analysis. Crit Care Med 44(5): 992-999.

16. Hu RF, Jiang XY, Chen J, Zeng Z, Chen XY, et al. (2015) Non-pharmacological interventions for sleep 
promotion in the intensive care unit. Cochrane Database Syst Rev (10): CD008808.

17. Mahran KSG, Abd-Elshafy KS, Abd El-hmeed AN, Ibraheem AIA, Abd-El Hafez GN, et al. (2018) The effect of night light on delirium occurrence in postoperative cardiac patients. IOSR Journal of Nursing and Health Science (IOSR-JNHS) 7(1): 1-7.

18. Richardson A, Allsop M, Coghill E, Turnock C (2007) Earplugs and eye masks: do they improve critical care patients' sleep? Nurs Crit Care 12: 278-286.

19. Hu RF, Jiang XY, Zeng YM, Chen XY, Zhang YH (2010) Effects of earplugs and eye masks on nocturnal sleep, melatonin and cortisol in a simulated intensive care unit environment. Crit Care 14(2): R66.

20. Scotto CJ, McClusky C, Spillan S, Kimmel J (2009) Earplugs improve patients' subjective experience of sleep in critical care. Nurs Crit Care 14(4): 180-184.

21. Bourne RS, Mills GH, Minelli C (2008) Melatonin therapy to improve nocturnal sleep in critically ill patients: encouraging results from a small randomised controlled trial. Crit Care 12(2): R52.

22. Joseph A (2018) Sedation of the trauma patient in the intensive care unit. Journal of Emergency and Critical Care Medicine 2: 8.

23. Kamdar B, Needham D, Collop NA (2012) Sleep deprivation in critical illness: its role in physical and psychological recovery. J Intensive Care Med 27(2): 97-111.

24. Van Rompaey B, Elseviers MM, Schuurmans MJ, Shortridge Baggett LM, Truijen S, et al. (2009) Risk factors for delirium in intensive care patients: a prospective cohort study. Crit Care 13(3): R77.

25. Inouye S (2006) Delirium in older persons. N Engl J Med 354(11): 1157-1165.

26. Xie J, Kang J, Mills G (2009) Clinical review: The impact of noise on patient's sleep and the effectiveness of noise reduction strategies in intensive care units. Crit Care 13(2): 208.

27. Ghaeli $\mathrm{P}$, Shahhatami F, Zade MM, Mohammadi M, Arbabi M (2018) Preventive Intervention to Prevent Delirium in Patients Hospitalized in Intensive Care Unit. Iran J Psychiatry 13(2): 142-147.

28. Estrup S, Kjer CKW, Poulsen LM, Gogenur I, Mathiesen O (2018) Delirium and effect of circadian light in the intensive care unit: a retrospective cohort study. Acta Anaesthesiologica Scand 62(3): 367-375.

29. Park M, Kohlrausch A, de Bruijn W, de Jager P, Simons K (2014) Analysis of the soundscape in an intensive care unit based on the annotation of an audio recording. J Acoust Soc Am 135(4): 1875-1886. 